\title{
Role of Bowel Preparation in Adenoma Detection Rate and Follow-up Recommendations in African American Dominant Patient Population
}

Hamid-Reza Moein ${ }^{1}$, Eskara Pervez ${ }^{2}$, Salina Faidhalla ${ }^{2}$, Heba Habbal ${ }^{3}$, Hajra Khan ${ }^{2}$, Anshu Wadehra ${ }^{2}$, Mahvish Khalid ${ }^{2}$, Diana Kakos ${ }^{4}$, Paul Naylor ${ }^{5}$, Bashar Mohamad ${ }^{6}$

1. Internal Medicine, Sinai-Grace Hospital/Detroit Medical Center, Detroit, USA 2. Internal Medicine, Wayne State University/Detroit Medical Center, Detroit, USA 3. Internal Medicine, Beaumont Hospital, Dearborn, USA 4. Internal Medicine, Wayne State University School of Medicine, Bloomfield Hills, USA 5. Gastroenterology, Wayne State University School of Medicine, Detroit, USA 6. Gastroenterology and Hepatology, Wayne State University, Detroit, USA

Corresponding author: Hamid-Reza Moein, moein.hr@gmail.com

\begin{abstract}
Introduction: Bowel preparation quality in colonoscopy is one of the most essential components of quality assessment. According to the latest guidelines, inadequate bowel preparation warrants repeat colonoscopy in less than a year. Our aim was to investigate the role of bowel preparation in adenoma detection rate (ADR), its relationship with patients' demographics, and compliance with subsequent surveillance recommendations with guidelines.
\end{abstract}

Methods: This is a retrospective chart review study. Bowel preparation quality was divided into three categories: high, intermediate, and low. ADR and polyp detection rates (PDR) were calculated as the number of patients with adenoma or polyp divided by the total number of patients.

Results: Among 1,062 patients (934 African American and 128 non-African American) 81\%, 11\%, and 8\% had high, intermediate, and low-quality bowel preparations, respectively. Race, gender, age, type of endoscopist, and body mass index did not play any role in bowel preparation quality. ADR and PDR were significantly higher in African Americans as compared to non-African Americans. ADR was significantly lower in the lowquality as compared to the high- and intermediate-quality bowel preparations $(\mathrm{OR}=2.13$; $\mathrm{p}=0.0032)$. Bowel preparation quality was not correlated with subsequent follow-up recommendations. Academic gastroenterologists and surgeons had the highest and lowest compliance with surveillance guidelines, respectively.

Conclusions: Racial and gender disparity appears to have no meaningful effect on the quality of bowel preparation. Only two categories (adequate [high/intermediate] or inadequate [low-quality]) may be used for follow-up recommendations. Non-compliance with surveillance guidelines is concerning and may inadvertently increase the interval risk of colorectal cancer.

Review began 06/15/2021 Review ended 06/30/2021 Published 06/30/2021

() Copyright 2021

Moein et al. This is an open access article distributed under the terms of the Creative Commons Attribution License CC-BY 4.0., which permits unrestricted use, distribution, and reproduction in any medium, provided the original author and source are credited.
Categories: Gastroenterology

Keywords: bowel preparation quality, adenoma detection rate, surveillance colonoscopy recommendations, screening colonoscopy, surveillance colonoscopy

\section{Introduction}

Colonoscopy is currently the gold standard of colorectal cancer (CRC) detection and also is used to prevent or reduce the risk of CRC by removing adenomatous polyps [1]. Quality metrics of screening and surveillance colonoscopies should be monitored routinely. Bowel preparation and adenoma detection rate (ADR) are the most important quality measures in colonoscopy [2,3]. Bowel preparation quality plays a crucial role in diagnostic accuracy and therapeutic safety of colonoscopy [3-5]. Inadequate bowel preparation can result in an estimated adenoma miss rate of $28 \%-42 \%$, prolonged procedure times, increased costs, and also increased adverse events [3,4]. According to the latest Standards of Practice Committee of the American Society for Gastrointestinal Endoscopy, in patients with inadequate bowel preparations repeat colonoscopy in less than a year is recommended [6].

Studies showed conflicting results regarding the correlation of bowel preparation quality and ADR [5,7-11]. The majority of studies demonstrated similar ADR among intermediate and high-quality bowel preps and significantly lower ADR in low-quality bowel preps [8]. Some studies did not find any correlation between ADR and bowel preparation [10-12]. Moreover, better bowel preparation is not always equal to better ADR [9].

Information in African American patient population regarding bowel preparation and ADR is lacking in the literature. Knowing that African Americans have the highest prevalence of colorectal adenoma [13] and the 
highest incidence and mortality rate from CRC [14], characterizing factors contributing to bowel preparation quality and its correlation with ADR are highly important in this patient population. Therefore, we aimed to investigate the correlation between bowel preparation and ADR in African American population and compare it to non-African Americans. Considering the well-known effect of body weight, gender, and age on ADR [10,11], we evaluated the relationship of these risk factors with bowel preparation. Furthermore, we sought to investigate the adherence of endoscopists to gastrointestinal society guidelines for follow-up colonoscopy recommendations.

\section{Materials And Methods Study population and measurements}

This is a retrospective, cross-sectional, chart review study. Consecutive electronic medical records of patients who underwent screening or surveillance colonoscopy at Harper Hospital/Detroit Medical Center's endoscopy suite (open access) in the last six months of 2017 were reviewed. Age, gender, body mass index (BMI), race, bowel preparation quality, number of polyps, adenoma detection, endoscopist specialty (Gastroenterologist vs. surgery), surveillance vs. screening colonoscopy, and follow-up recommendations were reviewed from electronic medical records. This study was approved by the Institutional Review Board of Wayne State University.

\section{Bowel preparation}

Drinking four liters of polyethylene glycol the day before colonoscopy was used as the bowel preparation regimen in all the patients. Bowel preparation quality was recorded based on physician reporting in the procedure notes. Most endoscopists used a modified Aronchick bowel preparation scale to describe the bowel preparation quality [15]. In brief, excellent quality is defined as $>95 \%$ of the mucosal surface seen; good quality is defined as a $>90 \%$ of the mucosal surface seen with a large volume of clear; fair quality is defined as some semisolid stool that could be suctioned or washed away but $>90 \%$ of the mucosal surface seen; poor quality indicates $<90 \%$ of the mucosal surface seen with semisolid stool that could not be suctioned or washed away; and insufficient quality indicates that fecal material could not be cleared.

Only one patient had a report based on Boston Bowel Preparation Scale, which was $>5$. That was considered equal to good prep on the Aronchick scale, according to a validation study [16]. For analysis purposes in this study, we divided the bowel preparations into three different groups: high quality (including excellent and good preparation), intermediate quality (fair preparation), and low quality (poor/insufficient preparation). In $12.9 \%$ of colonoscopies, the bowel preparation quality was not reported by physicians and in those cases, a category was chosen indirectly based on the other components of the endoscopy report such as comments related to visualization of the colon, small polyp detection, and follow-up recommendations.

\section{Colonoscopy and follow-up recommendations}

All colonoscopies were performed by certified academic and non-academic gastroenterologists (non-AGI) or surgeons. The follow-up interval recommendation for the next colonoscopy is obtained from the procedure notes written by endoscopists after their procedure. Then the follow-up recommendations were compared with the relevant available guidelines in 2017 based on the number of polyps or adenomas and quality of preparation $[4,6,17]$. In brief, guidelines indicate follow-up colonoscopy in less than a year for inadequate bowel preparation, three to five years for adenomas based on their number and size, and 10 years for hyperplastic or benign polyps.

\section{ADR and PDR calculation}

ADR was defined as the proportion of colonoscopies with at least one adenoma detected and is reported separately for each bowel preparation. Polyp detection rate (PDR) was calculated similarly as the number of examinations with at least one polyp was divided by the total number of examinations.

\section{Statistical analysis}

Statistical analysis was performed by JMP 14.0 Software (SAS Institute Inc., Cary, NC, USA). Descriptive data are presented as mean \pm SEM. Odds ratio (ORs) and $95 \%$ confidence intervals (CIs) were preferentially used for the comparisons of ADR and PDR. ANOVA and Chi-square tests were used to compare the quality of bowel preparation with other variables. Linear regression and Spearman test were used for correlations between bowel preparation and ADR with demographics. A P-value of $<0.05$ is considered significant.

\section{Results}

A total of 1,062 patients were included and analyzed in this study out of 1,095 reviewed electronic charts. Among those 33 excluded were six with a tumor, 13 with missing pathology or lost biopsy and 14 were aborted. The predominant study population were African Americans who constituted $87.9 \%$ of the patients. Non-African American population included Caucasians (8.5\%), Asians (1.6\%), Hispanics (0.84\%), American Indians ( $0.75 \%)$, and Middle Eastern (0.18\%). The mean age was $59 \pm 0.19$ years and $46.6 \%$ were males. 


\section{Cureus}

African American patients were statistically younger and had higher BMI as compared to non-African Americans (Table 1).

\begin{tabular}{|c|c|c|c|c|}
\hline & African American $(\mathrm{n}=934)$ & Non-African American $(n=128)$ & Total $(n=1,062)$ & $\mathbf{P}^{*}$ \\
\hline Age (year) & $59 \pm 0.2$ & $60 \pm 0.6$ & $59 \pm 0.2$ & 0.042 \\
\hline Gender (male \%) & $46 \%$ & $45 \%$ & $54 \%$ & 0.91 \\
\hline BMI (kg/m²) & $31 \pm 0.2$ & $30 \pm 0.7$ & $31 \pm 0.2$ & 0.02 \\
\hline Colonoscopy indication (screening \%) & $80 \%$ & $14 \%$ & $80 \%$ & 0.10 \\
\hline \multicolumn{5}{|l|}{ Bowel preparation quality } \\
\hline High (Excellent/Good) & $747(82 \%)$ & $108(84 \%)$ & $855(81 \%)$ & 0.4 \\
\hline Intermediate (Fair) & $107(12 \%)$ & $10(8 \%)$ & $117(11 \%)$ & 0.43 \\
\hline Low (Poor/Insufficient) & $80(8 \%)$ & $10(8 \%)$ & $90(8 \%)$ & 0.43 \\
\hline
\end{tabular}

TABLE 1: Demographics and bowel preparation quality among African American and non-African American patients.

BMI, Body mass index. *P-value comparing African Americas vs. non-African Americans using t-test or Chi-square tests.

The majority of colonoscopies were performed by AGI (66\%) followed by non-AGI/private gastroenterologists (17\%) and surgeons (17\%).

\section{Bowel preparation quality and demographics}

Bowel preparation quality is reported separately for African Americans and non-African Americans in Table 1. In total, $81 \%, 11 \%$, and $8 \%$ of patients had high, intermediate, and low-quality bowel preparations, respectively. There was no significant difference between African Americans and non-African Americans in respect to bowel preparation quality (Table 1). The role of demographics such as age, gender, and BMI that may play a role in the quality of bowel preparation was evaluated. Demographics of patients with different bowel preparation categories were similar (Table 2).

\begin{tabular}{|c|c|c|c|c|c|c|}
\hline \multirow{2}{*}{ Bowel preparation quality } & \multicolumn{3}{|c|}{ African American } & \multicolumn{3}{|c|}{ Non-African Americans } \\
\hline & Age & Male (\%) & BMI $\left(\mathrm{kg} / \mathrm{m}^{2}\right)$ & Age & Male (\%) & BMI $\left(\mathrm{kg} / \mathrm{m}^{2}\right)$ \\
\hline High & $58.3 \pm 0.2$ & $46 \%$ & $31 \pm 0.27$ & $59 \pm 0.16$ & $46 \%$ & $29.0 \pm 0.7$ \\
\hline Intermediate & $59.4 \pm 0.5$ & $51 \%$ & $31 \pm 0.73$ & $63.2 \pm 2.14$ & 30 & $31.5 \pm 2.3$ \\
\hline Low & $59.2 \pm 0.6$ & $50 \%$ & $32 \pm 0.84$ & $61 \pm 2.14$ & $50 \%$ & $28.7 \pm 2.3$ \\
\hline$P^{\star}$ & 0.17 & 0.31 & 0.73 & 0.16 & 0.58 & 0.62 \\
\hline
\end{tabular}

TABLE 2: Correlation of bowel preparation quality with demographics among African Americans and non-African Americans who had similar bowel preparation regimen.

BMI, Body mass index. *P-values representative of the difference between groups in each column.

Colonoscopy metrics are reported in Table 3. Optimal performance as defined by reaching the cecum was significantly lower in the low-quality bowel preparation as compared to the intermediate- and high-quality bowel preparations ( $p=0.0001$ ). Total mean number of found polyps were $1.2 \pm 0.0,1.2 \pm 0.1$, and $0.7 \pm 0.1$ in high, intermediate, and low-quality bowel preparations, respectively $(\mathrm{p}=0.028)$. The total PDR was significantly higher in high- and intermediate-quality bowel preparation as compared to low-quality bowel preparations $(\mathrm{p}=0.0005)$. Similarly, ADR was significantly higher in high- and intermediate-quality bowel preparations in comparison with low-quality bowel preparation ( $\mathrm{p}=0.01$ for both, respectively) (Table 3). ADR and PDR were similar in high- vs. intermediate-quality bowel preparation ( $p>0.05$ ) (Table 3). ADR was 
significantly higher in African American patients (38\%) compared with non-African American patients (27\%) ( $p=0.0213)$. Similarly, PDR was also higher in African Americans compared to non-African Americans (56\% vs. $46 \%$; $\mathrm{p}=0.0310)$.

\begin{tabular}{|c|c|c|c|c|c|}
\hline $\begin{array}{l}\text { Bowel } \\
\text { preparation } \\
\text { quality }\end{array}$ & $\begin{array}{l}\text { Cecum intubation } \\
\qquad(n=1,062)\end{array}$ & $\begin{array}{l}\text { Polyp detection rate } \\
\qquad(n=1,062)\end{array}$ & $\begin{array}{l}\text { Adenoma detection } \\
\text { rate } \quad(n=1,062)\end{array}$ & $\begin{array}{l}\text { ADR in screening } \\
\text { colonoscopies } \\
(n=846)\end{array}$ & $\begin{array}{l}\text { ADR in surveillance } \\
\text { colonoscopies } \\
(n=216)\end{array}$ \\
\hline High & $99 \%$ & $58 \%$ & $38 \%$ & $37 \%$ & $44 \%$ \\
\hline Intermediate & $98 \%$ & $49 \%$ & $38 \%$ & $33 \%$ & $52 \%$ \\
\hline Low & $86 \%$ & $38 \%$ & $22 \%$ & $18 \%$ & $39 \%$ \\
\hline $\mathbf{P}^{*}$ & 0.0001 & 0.0005 & 0.013 & 0.0078 & 0.65 \\
\hline
\end{tabular}

TABLE 3: Colonoscopy metrics as a function of bowel preparation quality.

ADR, Adenoma detection rate. *P values for columns calculated based on Pearson chi-square test.

\section{Follow-up recommendations and compliance of endoscopists with standard guidelines}

Among patients with low-quality bowel preparation (87 patients), 21.0\% did not have any follow-up recommendations (Table 4). When documented follow-up recommendations for low-quality bowel preparation were evaluated by specialty, AGI had more documentation (93\%) as compared to non-AGI (57\%) or surgeons (39\%) $(\mathrm{p}=0.0001)$. Surgeons had the lowest compliance with standard guidelines (22\%) as compared to non-AGI (59\%) and AGI $(80 \%)(\mathrm{p}=0.0001)$. Participation of fellows with AGI did not lead to a significant change in the percentage of follow-up recommendation documentation (93\%) or correct recommendations (80\%) as compared to the AGI alone (93\% and $80 \%$, respectively). We did not find any significant correlation between different group of endoscopists and bowel preparation quality $(\mathrm{p}=0.12)$.

\begin{tabular}{|l|l|l|l|l|}
\hline $\begin{array}{l}\text { Bowel preparation } \\
\text { quality }\end{array}$ & $\begin{array}{l}\text { Recommendation* is provided } \\
(\%)\end{array}$ & $\begin{array}{l}\text { Correct recommendation } \\
(\%)\end{array}$ & $\begin{array}{l}\text { Longer recommendation } \\
(\%)\end{array}$ & $\begin{array}{l}\text { Shorter recommendation } \\
\text { (\%) }\end{array}$ \\
\hline High & $78 \%$ & $73 \%$ & $2 \%$ & $25 \%$ \\
\hline Intermediate & $83 \%$ & $68 \%$ & $9 \%$ & $23 \%$ \\
\hline Low & $79 \%$ & $78 \%$ & $18 \%$ & $4 \%$ \\
\hline
\end{tabular}

TABLE 4: Guideline-directed follow-up recommendations based on the bowel preparation quality.

*Recommendations are based on Standards of Practice Committee of the American Society for Gastrointestinal Endoscopy published in 2015 [4].

There was no significant correlation between different bowel preparation categories and provided recommendations or recommendation compliance rates (Table 4). The most non-compliance with follow-up guidelines was observed in the intermediate-quality bowel preparation (32\%) as compared to low and highquality bowel preparations ( $22 \%$ and $27 \%$, respectively). This could reflect possible ambiguity in this small number of patients with respect to physician decision. Given that poor preparation quality should require a return within a year when a recommendation was given, $18 \%$ of the time it was for a longer interval than recommended by the guidelines (Table 4 ).

\section{Discussion}

Bowel preparation is one of the essential quality metrics in colonoscopy $[2,3]$. In this study, we specifically investigated bowel preparation and its role in detecting polyps and adenomas among African American dominant patient population, which data are limited. We found that adequate (high and intermediate) bowel preparation results in higher ADR as compared to low-quality bowel preparation ( $38 \%$ vs. $22 \%$, OR $=2.13$; $\mathrm{p}=0.0032$ ). The ADR gap between high- and intermediate- bowel preparation vs. low-quality bowel preparation was even higher in the screening colonoscopies ( $36 \%$ vs. $19 \%, \mathrm{OR}=2.54 ; \mathrm{p}=0.0023$ ). This is very important since ADR has a reverse correlation with CRC detection and death [2,18-20]. Similar to our study, Clark et al. [8] demonstrated that high- and intermediate-quality bowel preparations resulted in higher ADR 
as compared to low-quality bowel preparation ( $\mathrm{OR}=1.39)$. In addition, we did not find any significant difference in ADR among high- and intermediate (fair)-quality bowel preparation, which was similar to Clark et al. [8]. However, Menees et al. [21] demonstrated a 28\% adenoma miss rate in those patients with fair (intermediate-quality) bowel preparation when repeated colonoscopy in a three-year period. On the other hand, Park et al. [11] and Rai et al. [10] did not find any significant correlation between the quality of bowel preparation and ADR. Nevertheless, they found a trend and they attributed their finding to possibly nonsufficient sample size to detect the difference. Analysis from New Hampshire Colonoscopy Registry also did not find any significant correlation between different qualities of bowel preparation and ADR, which was consistent for both screening and surveillance colonoscopies [12]. In the same line, despite finding a trend towards lower ADR in surveillance colonoscopies with low-quality bowel preparation compared to adequate bowel preparation, there was no significant difference. This finding can be attributed to a low number of surveillance colonoscopies in this study (20\% of total colonoscopies) or longer withdrawal time in surveillance vs. screening colonoscopies (not recorded in this study). In addition, we showed significantly higher ADR and PDR in African Americans in comparison with non-African Americans. Similarly, Lebwohl et al. demonstrated significantly higher ADR and prevalence of adenoma and advanced adenoma in African Americans in comparison with Caucasians [13]. However, a recent review indicates mixed evidence in ADR and prevalence of adenomas among African Americans and Caucasians [22].

Inadequate bowel preparation has shown increased total cost of a colonoscopy, increased procedure time and adenoma missed rate [3,4]. Our data suggest that race itself does not play a role in the quality of bowel preparation. This is in accordance with Lebwohl et al. in which they did not find any significant difference in bowel preparation quality among different races after multivariant analysis [23]. On the other hand, Appannagari et al. reported African American race as an independent risk factor for inadequate bowel preparation [24]. They found 50\% increased risk of inadequate bowel preparation among African Americas as compared to whites despite controlling for education and income. Despite the statistically significant difference in age and BMI between African Americans and non-African Americans, in our study, gender, age, endoscopist's specialty, and BMI were not significantly correlated with bowel preparation quality. On contrary, other studies showed that obesity, advanced age, and male gender could be risk factors of poor bowel preparation $[10,11,23,25,26]$. However, Anderson et al. showed a significant correlation with younger age and poor bowel preparation [12]. We did not observe any significant difference in bowel preparation quality among different type of endoscopists (i.e., surgeons, AGI, and non-AGI). However, Sapci et al. demonstrated that lower bowel preparation quality among surgeons had a correlation with the lower ADR in that group of endoscopists in comparison with gastroenterologists [27]. One of the reasons for discrepancies between different studies and our study can be different patient populations and races. This study is comprised of African American dominant population but the above-mentioned studies [10-12,23-26] were either Caucasian or Asian dominant. Poor compliance with bowel preparation instructions, comorbidities such as diabetes mellitus and stroke, longer appointment wait times [4,25,26], smoking [11,12], procedure time (after 11 AM [23] or after 12 PM [24]), Medicaid insurers [23], and single status [23] were all have been associated with low-quality bowel preparation.

Follow-up colonoscopy recommendations in patients with low-quality bowel preparation were missing in $24.2 \%$ and were not compliant with guidelines in $16.7 \%$ of cases in our study. Menees et al. reported that only $13 \%$ of their endoscopists complied with current recommended guidelines [21]. On the other hand, Walker et al. showed 97\% compliance of a single-center endoscopy suite with follow-up colonoscopy guidelines [28]. Reasons for non-compliance for guidelines include knowledge gap, not agreeing with the current national guidelines, or just simply because it has not been recorded in the electronic records by the endoscopists. A national survey result indicates a knowledge gap among gastroenterologists about the current surveillance guidelines [29]. This survey in 2004 showed that only $78 \%$ were familiar with the current national guidelines for surveillance colonoscopy and up to $76 \%$ disagreed with the guidelines [29]. A more updated and comprehensive investigation is warranted to evaluate the reasons for non-compliance of gastroenterologists or surgeons with national guidelines.

One of the strengths of this study is that all the included patients used the same bowel preparation regimen. This helped in making the comparisons more accurate and also allowed us to investigate other risk factors in bowel preparation quality with less bias. Moreover, this study is unique because of the African American dominant patient population, a population in which the current literature is limited. Our study had some limitations. Non-African American patient population sample size was smaller than African Americans, which might have decreased the power for detection of any difference among these two groups. Due to a limited number of patients of other races including Asian and Hispanic, we combined Caucasians and other races except for African American into one group of non-African American. In addition, $12.9 \%$ of endoscopists did not record the bowel preparation quality and in those cases, we concluded the bowel prep quality based on their findings and follow-up recommendations, which might not have been exactly accurate. Patients' compliance with bowel preparation instructions and follow-up recommendations, socioeconomic status, education, marital status, insurance, and time of the endoscopy (which were reported in some studies as risk factors for suboptimal bowel preparation) were not recorded in this study. Our results are limited to out-patient setting and in an African American dominant patient population and may not be generalizable. 


\section{Conclusions}

In conclusion, two categories of bowel preparation (i.e., adequate or inadequate) appear relevant for follow up recommendations after colonoscopy. The current study indicates that only inadequate or low-quality bowel preparation was associated with lower ADR. We did not observe any significant correlation between age, gender, race, and BMI with bowel preparation quality in this African American dominant patient population. Large prospective studies in African Americans are warranted to untangle the main risk factors for low-quality bowel preparation in this patient population and to address the conflicting results. Important quality measures such as recording follow-up recommendations and guideline-appropriate recommendations should be emphasized as when these measures are missing the risk of interval CRC may increase.

\section{Additional Information \\ Disclosures}

Human subjects: Consent was obtained or waived by all participants in this study. Wayne State University Institutional Review Board issued approval 096514M1E. No informed consent was needed for this study. Animal subjects: All authors have confirmed that this study did not involve animal subjects or tissue. Conflicts of interest: In compliance with the ICMJE uniform disclosure form, all authors declare the following: Payment/services info: All authors have declared that no financial support was received from any organization for the submitted work. Financial relationships: All authors have declared that they have no financial relationships at present or within the previous three years with any organizations that might have an interest in the submitted work. Other relationships: All authors have declared that there are no other relationships or activities that could appear to have influenced the submitted work.

\section{References}

1. Winawer SJ, Zauber AG, Ho MN, et al.: Prevention of colorectal cancer by colonoscopic polypectomy. The National Polyp Study Workgroup. N Engl J Med. 1993, 329:1977-81. 10.1056/NEJM199312303292701

2. Gurudu SR, Ramirez FC: Quality metrics in endoscopy. Gastroenterol Hepatol (N Y). 2013, 9:228-33.

3. Rex DK, Schoenfeld PS, Cohen J, et al.: Quality indicators for colonoscopy. Am J Gastroenterol. 2015, 110:72-90. 10.1038/ajg.2014.385

4. Saltzman JR, Cash BD, Pasha SF, et al.: Bowel preparation before colonoscopy. Gastrointest Endosc. 2015, 81:781-94. 10.1016/j.gie.2014.09.048

5. Guo R, Wang YJ, Liu M, et al.: The effect of quality of segmental bowel preparation on adenoma detection rate. BMC Gastroenterol. 2019, 19:119. 10.1186/s12876-019-1019-8

6. Levin B, Lieberman DA, McFarland B, et al.: Screening and surveillance for the early detection of colorectal cancer and adenomatous polyps, 2008: a joint guideline from the American Cancer Society, the US MultiSociety Task Force on Colorectal Cancer, and the American College of Radiology. CA Cancer J Clin. 2008, 58:130-60. 10.3322/CA.2007.0018

7. Kaminski MF, Wieszczy P, Rupinski M, et al.: Increased rate of adenoma detection associates with reduced risk of colorectal cancer and death. Gastroenterology. 2017, 153:98-105. 10.1053/j.gastro.2017.04.006

8. Clark BT, Rustagi T, Laine L: What level of bowel prep quality requires early repeat colonoscopy: systematic review and meta-analysis of the impact of preparation quality on adenoma detection rate. Am J Gastroenterol. 2014, 109:1714-23; quiz 1724. 10.1038/ajg.2014.232

9. Calderwood AH, Thompson KD, Schroy PC 3rd, Lieberman DA, Jacobson BC: Good is better than excellent: bowel preparation quality and adenoma detection rates. Gastrointest Endosc. 2015, 81:691-699.e1. 10.1016/j.gie.2014.10.032

10. Rai T, Navaneethan U, Gohel T, et al.: Effect of quality of bowel preparation on quality indicators of adenoma detection rates and colonoscopy completion rates. Gastroenterol Rep (Oxf). 2016, 4:148-53. 10.1093/gastro/gov002

11. Park JH, Kim SJ, Hyun JH, et al.: Correlation between bowel preparation and the adenoma detection rate in screening colonoscopy. Ann Coloproctol. 2017, 33:93-8. 10.3393/ac.2017.33.3.93

12. Anderson JC, Butterly LF, Robinson CM, Goodrich M, Weiss JE: Impact of fair bowel preparation quality on adenoma and serrated polyp detection: data from the New Hampshire colonoscopy registry by using a standardized preparation-quality rating. Gastrointest Endosc. 2014, 80:463-70. 10.1016/j.gie.2014.03.021

13. Lebwohl B, Capiak K, Neugut AI, Kastrinos F: Risk of colorectal adenomas and advanced neoplasia in Hispanic, black and white patients undergoing screening colonoscopy. Aliment Pharmacol Ther. 2012, 35:1467-73. 10.1111/j.1365-2036.2012.05119.x

14. Augustus GJ, Ellis NA: Colorectal cancer disparity in African Americans: risk factors and carcinogenic mechanisms. Am J Pathol. 2018, 188:291-303. 10.1016/j.ajpath.2017.07.023

15. Aronchick CA, Lipshutz WH, Wright SH, Dufrayne F, Bergman G: A novel tableted purgative for colonoscopic preparation: efficacy and safety comparisons with Colyte and Fleet Phospho-Soda. Gastrointest Endosc. 2000, 52:346-52. 10.1067/mge.2000.108480

16. Lai EJ, Calderwood AH, Doros G, Fix OK, Jacobson BC: The Boston bowel preparation scale: a valid and reliable instrument for colonoscopy-oriented research. Gastrointest Endosc. 2009, 69:620-5. 10.1016/j.gie.2008.05.057

17. Winawer SJ, Zauber AG, Fletcher RH, et al.: Guidelines for colonoscopy surveillance after polypectomy: a consensus update by the US Multi-Society Task Force on Colorectal Cancer and the American Cancer Society. Gastroenterology. 2006, 130:1872-85. 10.1053/j.gastro.2006.03.012

18. Corley DA, Jensen CD, Marks AR, et al.: Adenoma detection rate and risk of colorectal cancer and death . N Engl J Med. 2014, 370:1298-306. 10.1056/NEJMoa1309086 
19. Rex DK, Petrini JL, Baron TH, et al.: Quality indicators for colonoscopy. Am J Gastroenterol. 2006, 101:87385. 10.1111/j.1572-0241.2006.00673.x

20. Click B, Pinsky PF, Hickey T, Doroudi M, Schoen RE: Association of colonoscopy adenoma findings with long-term colorectal cancer incidence. JAMA. 2018, 319:2021-31. 10.1001/jama.2018.5809

21. Menees SB, Kim HM, Elliott EE, Mickevicius JL, Graustein BB, Schoenfeld PS: The impact of fair colonoscopy preparation on colonoscopy use and adenoma miss rates in patients undergoing outpatient colonoscopy. Gastrointest Endosc. 2013, 78:510-6. 10.1016/j.gie.2013.03.1334

22. Rutter CM, Knudsen AB, Lin JS, Bouskill KE: Black and white differences in colorectal cancer screening and screening outcomes: a narrative review. Cancer Epidemiol Biomarkers Prev. 2021, 30:3-12. 10.1158/10559965.EPI-19-1537

23. Lebwohl B, Wang TC, Neugut AI: Socioeconomic and other predictors of colonoscopy preparation quality . Dig Dis Sci. 2010, 55:2014-20. 10.1007/s10620-009-1079-7

24. Appannagari A, Mangla S, Liao C, Reddy KG, Kupfer SS: Risk factors for inadequate colonoscopy bowel preparations in African Americans and whites at an urban medical center. South Med J. 2014, 107:220-4. 10.1097/SMJ.0000000000000087

25. Mahmood S, Farooqui SM, Madhoun MF: Predictors of inadequate bowel preparation for colonoscopy: a systematic review and meta-analysis. Eur J Gastroenterol Hepatol. 2018, 30:819-26.

10.1097/MEG.0000000000001175

26. Wong MCS, Ching JYL, Chan VCW, et al.: Determinants of bowel preparation quality and its association with adenoma detection: a prospective colonoscopy study. Medicine (Baltimore). 2016, 95:e2251. 10.1097/MD.0000000000002251

27. Sapci I, Aiello A, Gorgun E, Rizk M, Delaney CP, Steele SR, Valente MA: Screening colonoscopy: high quality regardless of endoscopist specialty. Am J Surg. 2019, 217:442-4. 10.1016/j.amjsurg.2018.09.009

28. Walker AS, Nelson DW, Fowler JJ, et al.: An evaluation of colonoscopy surveillance guidelines: are we actually adhering to the guidelines?. Am J Surg. 2013, 205:618-22. 10.1016/j.amjsurg.2012.12.006

29. Saini SD, Nayak RS, Kuhn L, Schoenfeld P: Why don't gastroenterologists follow colon polyp surveillance guidelines?: results of a national survey. J Clin Gastroenterol. 2009, 43:554-8.

10.1097/MCG.0b013e31818242ad 